\title{
New Classification of Gastric Varices: A Twenty-Year Experience
}

\author{
Akash Singh ${ }^{1} \quad$ Nipun Verma ${ }^{1} \quad$ Sahaj Rathi ${ }^{1} \quad$ Sunita Kumari ${ }^{1} \quad$ Shivani Chandel ${ }^{2} \quad$ Virendra Singh ${ }^{1, \odot}$ \\ ${ }^{1}$ Department of Hepatology, Postgraduate Institute of Medical \\ Education and Research, Chandigarh, Punjab, India \\ 2Department of Internal Medicine, Postgraduate Institute of \\ Medical Education and Research, Chandigarh, Punjab, India \\ Address for correspondence Virendra Singh, MD, DM, FASGE, \\ Department of Hepatology, PGIMER, Chandigarh 160012, Punjab, \\ India (e-mail: virendrasingh100@hotmail.com).
}

J Digest Endosc 2021;12:24-30.

\begin{abstract}
Keywords

- gastric varices

- portal hypertension

Objectives Gastric varices (GV) have been classified by the Sarin classification for over two decades. However, a subset of these varices does not fit into this classification. We share our experience on GV in past 20 years and propose a modification.

Materials and Methods Consecutive patients with portal hypertension (PHT) posted for esophagogastroduodenoscopy were screened for GV between 1996 and 2016 at a tertiary-care hospital. GV were categorized as gastroesophageal varices (GOV1, GOV2) and isolated gastric varices (IGV1, IGV2) by Sarin's classification. Patients with varices in esophagogastric region as well as distally in the stomach or duodenum, thus having efferent drainage into the superior as well as inferior vena cava simultaneously, remain unclassified and were coined as GOV3.

Statistical Analysis Descriptive data was represented as mean (standard deviation) or median (interquartile range) or number (percentage). Chi-squared test, $t$-test, and logistic regression were done to compare groups and identify outcomes of interest.

Results GV were recognized in $400(11.5 \%)$ of 3,476 patients with PHT. Underlying disease was cirrhosis in 301 (75.2\%), extrahepatic portal venous obstruction in 78 (19.5\%), noncirrhotic portal fibrosis in 18 (4.5\%) and Budd-Chiari syndrome in 3 (0.75\%) patients with GV. GOV1, GOV2, IGV1, IGV2, and combined GOV1 with GOV2 were seen in 170 (42.5\%), 154 (38.5\%), 17 (4.3\%), 5 (1.3\%), and 12 (3.0\%) patients; respectively. GOV3 were identified in 42 (10.5\%) patients. Ninety-three patients with GV presented with gastrointestinal bleed and frequency of GOV2 was higher and GOV1 and GOV3 were lower among bleeders than non-bleeders.

Conclusions A significant proportion of patients with GV remain uncategorized by current classification among PHT patients. Addition of GOV3 in Sarin's classification will make it more comprehensive, uniform, and reproducible for future studies.
\end{abstract}

\section{Introduction}

Gastric varices (GV) are subepithelial portosystemic collaterals in the stomach that evolve as a consequence of portal hypertension (PHT). They are seen in around $20 \%$ of all patients with cirrhosis. ${ }^{1}$ Bleeding from GVs comprises 10 to $30 \%$ all variceal hemorrhages. These bleeds tend to be more published online September 28, 2020
DOI https://doi.org/

$10.1055 / \mathrm{s}-0041-1728222$ ISSN 0976-5042
(C)2020. Society of Gastrointestinal Endoscopy of India.

This is an open access article published by Thieme under the terms of the Creative Commons Attribution-NonDerivative-NonCommercial-License, permitting copying and reproduction so long as the original work is given appropriate credit. Contents may not be used for commercial purposes, or adapted, remixed, transformed or built upon. (https://creativecommons.org/licenses/by-nc-nd/4.0/).

Thieme Medical and Scientific Publishers Pvt. Ltd. A-12, 2nd Floor, Sector 2, Noida-201301 UP, India 
severe, and have higher mortality rates than bleeding from esophageal varices. ${ }^{1}$

GV are heterogenous in terms of their location, morphology as well as risk of bleeding. Various systems of classification (-Table 1) have been described for characterization of GVs, mainly based on location. ${ }^{1-5}$ Only the Hashizume classification takes into account the size and presence of warning signs of rupture. ${ }^{2}$ However, due to its complexity, this system is not very popular.

The mandate of a classification system of GVs is thus limited to description of the location of the varices in the stomach, which, in turn, dictates the drainage pathway. The classification by Sarin et al described over two decades back has globally been the most popular way of categorizing GVs. ${ }^{1}$ It is simple to apply, reproduce, and interpret for most cases of GVs, while also indicating the possible drainage pathway of the varices. It has been endorsed by Baveno-VI guidelines for PHT and variceal bleeding. ${ }^{6}$ Despite its many merits, there is a subset of cases that still remain uncategorized. We here propose an amendment in this classification based on our experience in past 20 years. It would make the classification more comprehensive and reduce semantic confusion while maintaining the physiologic description of GV.

\section{Materials and Methods}

An observational-analytic study was conducted between 1996 and 2016, where consecutive patients with PHT presenting to the Department of Hepatology at a tertiary-care institute for esophagogastroduodenoscopy (EGD) were recruited and their data was prospectively entered in the record books. The institute caters to more than 40,000 liver patients in a year from more than eight states in the country. Patients with severe cardiorespiratory disease, age $<18$ years, and human immunodeficiency virus infection were excluded. The patients were evaluated for the presence of varices and findings were noted on a standardized reporting sheet. The records were analyzed retrospectively to identify the proportion of patients with esophageal, gastric, gastroesophageal varices (GOV), and ectopic varices. The grade, location, and stigmata of recent bleed were noted. Procedures done during EGD including band ligation, sclerosant, or glue injection were recorded. Clinical details of recruited patients were retrieved from hospital medical records. Informed consent for EGD was taken from all patients and study was performed as per the good clinical practice guidelines, and Declaration of Helsinki.

Variables: PHT was defined on imaging (dilated portal vein, splenomegaly, and collaterals), endoscopic (varices and

Table1 Classification systems for gastric varices

\begin{tabular}{|c|c|}
\hline \multicolumn{2}{|c|}{ A. Sarin's classification of gastric varices ${ }^{1}$} \\
\hline $\begin{array}{l}\text { Gastroesophageal varices } \\
\text { GOV1 } \\
\text { GOV2 }\end{array}$ & $\begin{array}{l}\text { Varices in continuity with esophageal varices } \\
\text { Along the lesser curvature } \\
\text { Along the greater curvature extending toward the gastric fundus }\end{array}$ \\
\hline $\begin{array}{l}\text { Isolated gastric varices } \\
\text { IGV1 } \\
\text { IGV2 }\end{array}$ & $\begin{array}{l}\text { Isolated cluster of gastric varices in the gastric fundus } \\
\text { Isolated gastric varices in the other parts of the stomach }\end{array}$ \\
\hline \multicolumn{2}{|c|}{ B. Hashizume classification of gastric varices ${ }^{2}$} \\
\hline Form & F1 (tortuous), F2 (nodular) and F3 (tumorous) \\
\hline Location & La (anterior), Lp (posterior), LI (lesser curvature), Lg (greater curvature), Lf (fundus) \\
\hline Color & Cw (white), $\mathrm{Cr}$ (red) \\
\hline RCS & Glossy, thin-walled focal redness on the varix \\
\hline \multicolumn{2}{|c|}{ C. Hoskins and Johnson's classification of gastric varices ${ }^{3}$} \\
\hline Type 1 & Inferior extension of esophageal varices across the squamo-columnar junction \\
\hline Type 2 & Gastric varices located in fundus, which appear to converge to cardia with esophageal varices \\
\hline Type 3 & Gastric varices in fundus or body in the absence of esophageal varices \\
\hline \multicolumn{2}{|c|}{ D. Arkawa classification of gastric varices ${ }^{4}$} \\
\hline $\begin{array}{l}\text { Type I } \\
\text { la } \\
\text { lb }\end{array}$ & $\begin{array}{l}\text { A single supplying vessel forms a fundic varix } \\
\text { Plural supplying vessels join and form a varix that drains into a single vessel }\end{array}$ \\
\hline Type II & Gastric varices with multiple communications with vessels in stomach wall \\
\hline \multicolumn{2}{|c|}{ E. Mathur's classification of gastric varices ${ }^{5}$} \\
\hline Type 1 & Esophageal varices with lesser curvature varices \\
\hline Type 2 & Esophageal varices with fundal varices (2a-subcardiac and 2b-diffuse fundal) \\
\hline Type 3 & Isolated fundal varix (3a-due to splenic vein thrombosis, 3b-due to generalized portal hypertension) \\
\hline Type 4 & Lesser curvature gastric varices with esophageal varices with fundal varices \\
\hline Type 5 & Antral varices \\
\hline
\end{tabular}

Abbreviations: GOV, gastroesophageal varices; IGV, isolated gastric varices; RCS, red color spot. 
portal gastropathy) and clinical findings (spider nevi, splenomegaly, and relevant setting). Etiology of PHT was classified as cirrhosis, based on clinical (ascites, jaundice, edema, spider nevi), laboratory (low albumin, elevated prothrombin time, low platelet count), imaging (irregular outline and volume redistribution with signs of PHT), transient elastography, and biopsy findings; noncirrhotic portal fibrosis (NCPF) based on PHT with patent dilated splenoportal axis, normal liver stiffness, and absence of cirrhosis on biopsy ${ }^{7}$ extrahepatic portal vein obstruction (EHPVO) ${ }^{8}$ based on PHT with evidence of portal cavernoma; and Budd-Chiari syndrome (BCS) based on imaging findings blocked inferior vena cava (IVC) and/or hepatic veins. ${ }^{9}$

Classification of varices: GV were classified into gastroesophageal varices (GOV 1 and GOV 2) and isolated gastric varices (IGV 1 and IGV 2) as per Sarin's classification based on their location and drainage. ${ }^{1}$ We added a new class, GOV3, which included a combination of varices with efferent drainage to both the superior (SVC) and IVC system simultaneously. Therefore, presence of varices around the gastroesophageal junction- esophageal varices/GOV1/GOV2 (a majority of which drain through the periesophageal-azygous-superior vena cava pathway) in combination with antral and duodenal varices (draining into the posterior gastric-gastrospleno renal-inferior vena cava pathway) was called GOV3 (-Figs. 1 and 2). Esophageal varices were classified into five grades as described by Paquet, ${ }^{10}$ and grades 1 and 2 were considered small varices, and grades 3 and 4 large varices.

Diagnosis of GV: The varices were diagnosed based on endoscopic appearance of dilated venous channel with blue color on maximum and sustained air insufflation, done or supervised by a senior endoscopist with more than 30-year experience (VS). The presence of IGV2 in the body, antrum or duodenum of stomach, was concurred with another independent endoscopist. The varices were labeled as (a) antral varix: when a single dilated venous column, linear or polypoidal, is present in the antrum of the stomach, reaching the pyloric rim and persisting despite full inflation of air in the stomach, (b) duodenal varix: when one or more distinct, persistent, elevated venous columns are present in the first or second part of duodenum, running longitudinally or irregularly in the duodenum, (c) GV in body: when one or multiple column of dilated venous channels are seen in body of stomach sparing fundus and antrum.

Bleeding from GV: Gastric varices were considered to have bled if active bleeding, oozing, adherent clot, brownish ulceration, or cherry red spots were seen on the gastric varix or if there were large GV in a patient with a history of upper gastrointestinal bleed without esophageal varices or other detectable causes such as portal hypertensive gastropathy, gastric mucosal lesions, peptic ulceration, or gastric antral vascular ectasia. ${ }^{1}$

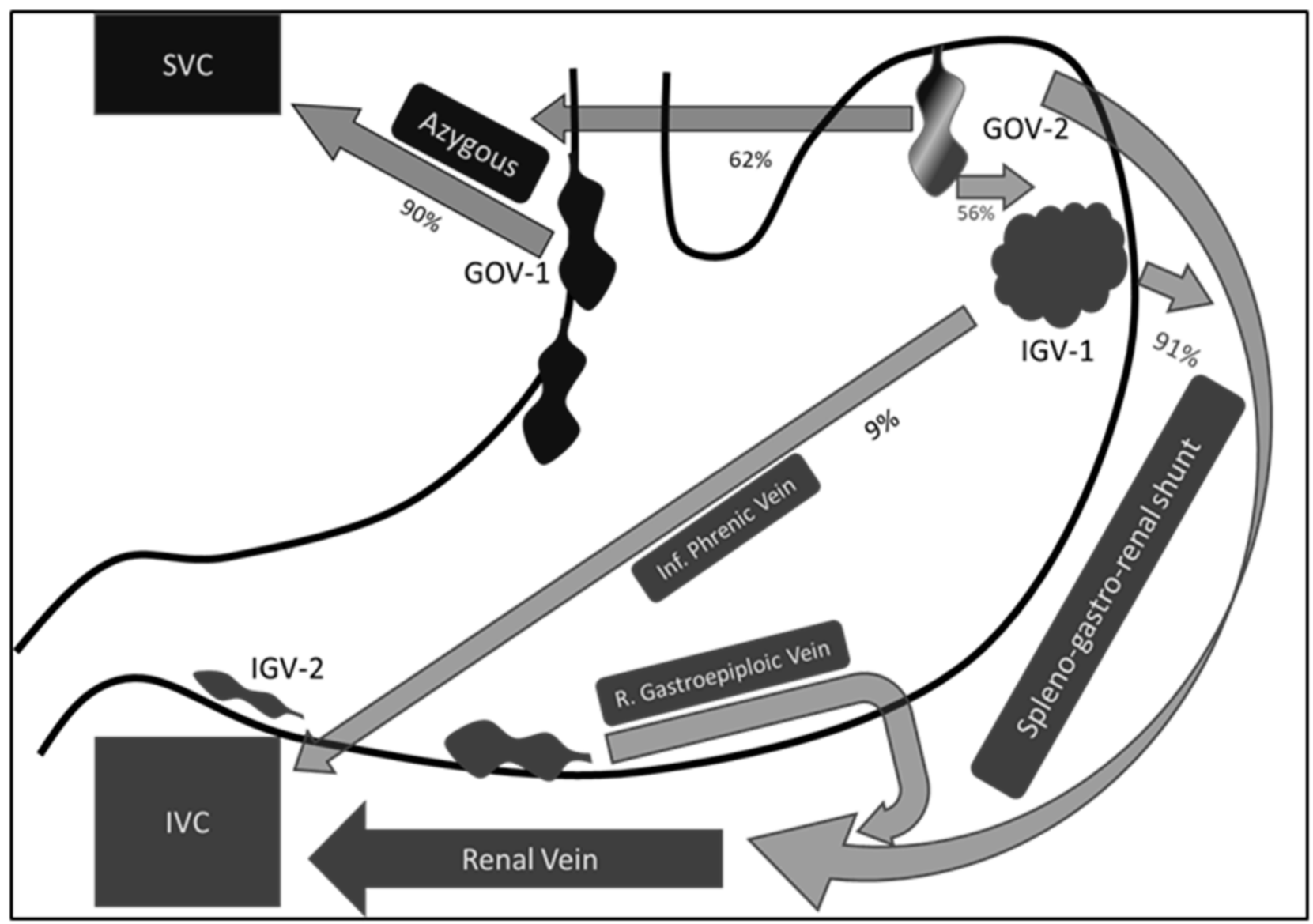

Fig. 1 Predominant drainage pathways of gastroesophageal varices. GOV, gastroesophageal varices; IGV, isolated gastric varices; IVC, inferior vena cava; SVC, superior vena cava. 


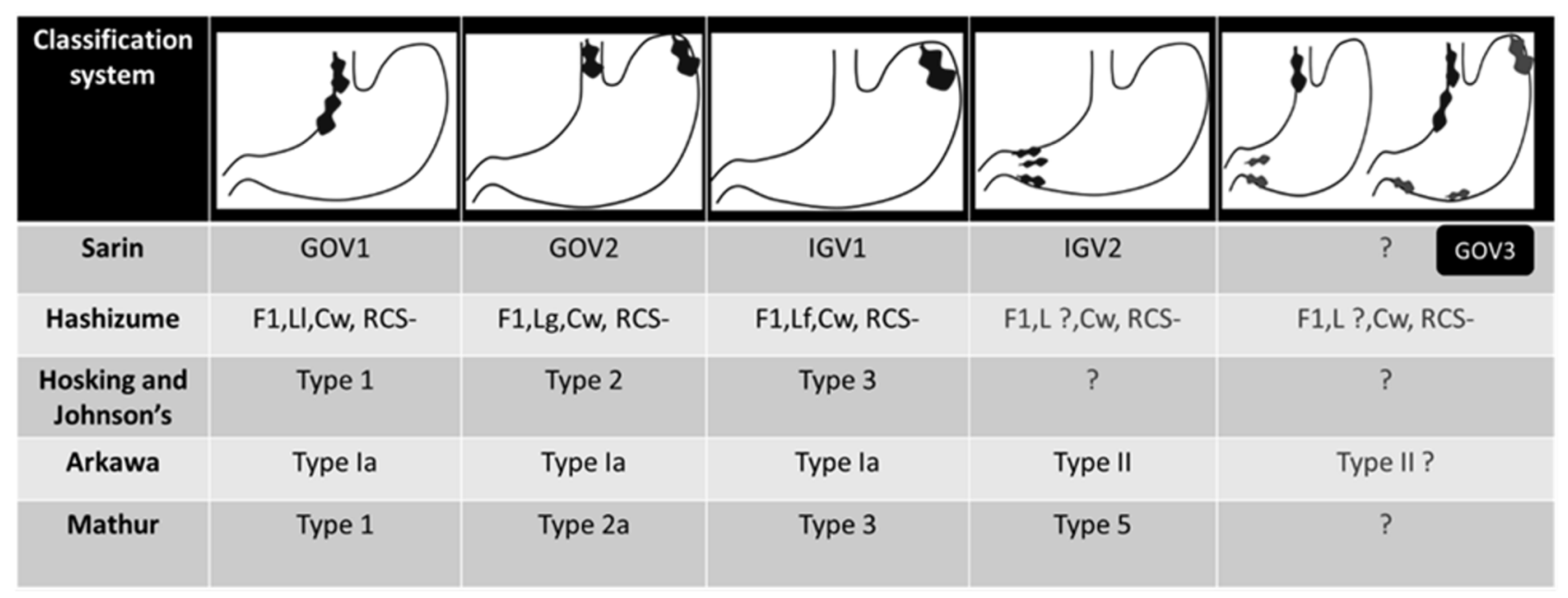

Fig. 2 Proposed new classification of gastric varices. "?” denotes inability to categorize varices with a particular classification. GOV, gastroesophageal varices; IGV, isolated gastric varices; IVC, inferior vena cava; RCS, red color spot; SVC, superior vena cava.

Management of bleeding from GV: After initial resuscitation with hydration, airway support, intravenous antibiotics and vasoactive agents, the GV bleed was managed with either endoscopic sclerotherapy with absolute alcohol or sodium tetradecyl sulfate or endoscopic variceal obturation with glue injection.

\section{Statistical Analysis}

Numerical data was analyzed as mean with standard deviation (SD) when normally distributed, and median with interquartile range when not-normally distributed. Categorical data was represented as number with proportions. Proportions between the two groups were compared using chi-squared or Fisher's exact test, and t-test or u-test depending on their applicability. Logistic regression was done for relationship between variables. A $p$-value of $<0.05$ (two-tailed) was considered as significant. The Statistical Package for Social Sciences (version 22.0 for Windows, SPSS Inc., Chicago, Illinois, United States) was used for analysis.

\section{Results}

Out of 3,476 patients with PHT who underwent EGD, 400 patients (11.4\%) had varices in the stomach or duodenum. Patients with GV presented at a mean age of 42.7 (SD: $15.7)$ years and were predominantly males (299, 74.7\%) (-Table 2). Etiology of PHT among patients with GV was cirrhosis in 301 (75.2\%), EHPVO in 78 (19.5\%), NCPF in 18 (4.5\%), and BCS in $3(0.75 \%)$ patients. Among patients with cirrhosis, 33 patients (10.9\%) had ascites, 4 patients (1.3\%) had hepatic encephalopathy, and 21 patients $(6.9 \%)$ had jaundice. Diabetes and hypertension were present in 4 (1.0\%) and 40 patients $(10.0 \%)$, respectively.

The distribution of GV as per Sarin's classification was GOV1 in 170 patients (42.5\%), GOV2 in 154 patients (38.5\%), IGV1 in 17 patients (4.3\%), IGV2 in 5 patients (1.3\%), and combined GOV1 with GOV2 in 12 patients (3.0\%). Forty-two patients $(10.5 \%)$ were not classified by Sarin's classification and were labeled GOV3. The distribution of GV including GOV1, 2, IGV and GOV3, mechanistic drainage, and classification scheme was described in - Figs. 1 to 3 . Patients with GOV3 had esophageal varices with antral varices $(3.3 \%)$ or duodenal varices (1.5\%), GOV1 with antral varices (2.3\%), GOV2 with antral varices (1.5\%), and combined GOV1 with

Table 2 Baseline characteristics of patients with gastric varices in the study population

\begin{tabular}{|c|c|c|}
\hline Sr. no. & Parameters & $n=400$ \\
\hline 1 & Age & $42.7(15.7)$ \\
\hline 2 & Gender (males) & $299(74.7 \%)$ \\
\hline \multirow[t]{5}{*}{3} & \multicolumn{2}{|c|}{ Etiology of portal hypertension } \\
\hline & Cirrhosis & 301 (75.2\%) \\
\hline & EHPVO & 78 (19.5\%) \\
\hline & NCPF & $98(4.5 \%)$ \\
\hline & BCS & $3(0.8 \%)$ \\
\hline \multirow[t]{11}{*}{4} & \multicolumn{2}{|l|}{ Investigations } \\
\hline & Hemoglobin-g/dL & $10.0(8.6-11.9)$ \\
\hline & Platelet count $-\times 10^{9} / \mathrm{L}$ & $83(63-143)$ \\
\hline & TLC-per mm³ & $5400(3,700-7120)$ \\
\hline & Serum creatinine-mg/dL & $0.83(0.36-1.10)$ \\
\hline & Bilirubin-mg/dL & $1.7(1.0-2.7)$ \\
\hline & ALT-U/L & $34.5(26.0-59.1)$ \\
\hline & ALP-U/L & $165(102-246)$ \\
\hline & Protein-g/dL & $6.9(6.4-7.8)$ \\
\hline & Albumin-g/dL & $3.6(2.9-4.2)$ \\
\hline & INR & $1.25(1.10-1.38)$ \\
\hline
\end{tabular}

Abbreviations: ALP, alkaline phosphatase; ALT, alanine aminotransferase; BCS, Budd-Chiari syndrome; EHPVO, extrahepatic portal venous obstruction; INR, international normalized ratio; NCPF, noncirrhotic portal fibrosis; TLC, total leucocyte count.

Data was represented as number (\%) or median (interquartile range) or mean (standard deviation). 


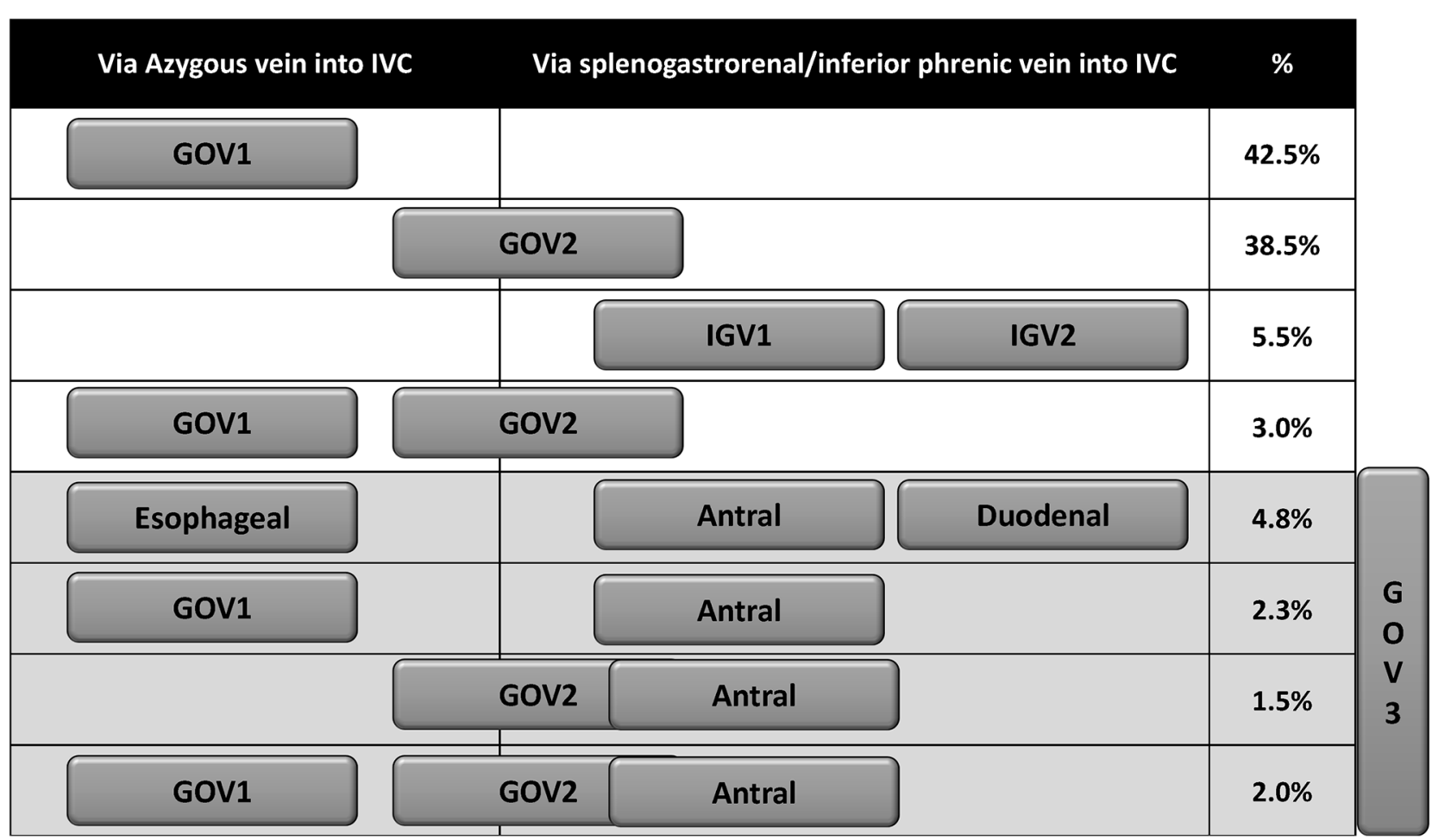

Fig. 3 Distribution of gastric varices in our population depicting efferent drainage. Shaded areas denote dual drainage varices (GOV3). GOV 2 can drain into IVC (44\%), SVC (38\%), or both (18\%). GOV, gastroesophageal varices; IGV, isolated gastric varices; IVC, inferior vena cava; SVC, superior vena cava.

Table 3 Characteristics of gastric variceal bleeders compared with nonbleeders

\begin{tabular}{|c|c|c|c|}
\hline Parameters & Bleeders $(n=93)$ & Nonbleeders $(n=307)$ & $p$-Value \\
\hline Age (years) & $42.0 \pm 14.2$ & $42.9 \pm 16.1$ & 0.622 \\
\hline Gender (M:F) & $74: 19$ & $225: 82$ & 0.222 \\
\hline \multicolumn{4}{|l|}{ Etiology of PHT } \\
\hline Cirrhosis & $63(67.7 \%)$ & $238(77.5 \%)$ & \multirow[t]{4}{*}{0.099} \\
\hline EHPVO & $26(28.0 \%)$ & $52(16.9 \%)$ & \\
\hline NCPF & $4(4.3 \%)$ & $14(4.6 \%)$ & \\
\hline BCS & $0(0.0 \%)$ & $3(1.0 \%)$ & \\
\hline \multicolumn{4}{|c|}{ Type of gastric varices } \\
\hline GOV1 & $31(33.3 \%)$ & $152(49.5 \%)$ & 0.006 \\
\hline GOV2 & $56(60.2 \%)$ & $111(36.2 \%)$ & $<0.001$ \\
\hline GOV3 & $3(3.2 \%)$ & $38(12.4 \%)$ & 0.011 \\
\hline IGV1 & $3(3.2 \%)$ & $11(3.6 \%)$ & 0.230 \\
\hline IGV2 & $0(0.0 \%)$ & $5(1.6 \%)$ & 0.216 \\
\hline
\end{tabular}

Abbreviations: BCS, Budd-Chiari syndrome; EHPVO, extrahepatic portal venous obstruction; F, female; GOV, gastroesophageal varices; ICV, isolated gastric varices; M, male; NCPF, noncirrhotic portal fibrosis; PHT, portal hypertension.

Data represented as mean (standard deviation) and number (\%).

GOV2 with antral varices (2.0\%). Out of 336 patients with GOV, 199 (59.2\%) patients had small esophageal varices, while 137 (40.7\%) had large esophageal varices.

Among patients with GVs, 93 (23.5\%) patients presented with gastrointestinal bleed. There was no difference in mean age, gender, and etiology of cirrhosis in bleeders and nonbleeders ( - Table 3 ). As compared with nonbleeders, bleeders had a higher frequency of GOV2 (odds ratio [OR]: 2.04, 95\% confidence interval [CI]: 1.27-3.29; $p<0.001$ ), lower GOV1 (OR: $0.43,95 \%$ CI: $0.26-0.69] ; p=0.006$ ), and lower GOV3 (OR: $0.35,95 \%$ CI: $0.15-0.80 ; p=0.011$ ). There was no difference in the frequencies of isolated GV in bleeders and nonbleeders. There was no relationship between presence of GOV3 and age, gender, etiology, or grade of esophageal 
varices. Bleeding due to GV was managed with either sclerotherapy with absolute alcohol or sodium tetradecyl sulfate in 18 (19.4\%) patients and glue injection in 75 (80.6\%) patients.

\section{Discussion}

In the present study, GV were found in $11.5 \%$ adult patients with PHT. GV had been reported heterogeneously (2-100\%) in literature possibly due to heterogenous selection criteria, referral bias, and diagnostic criteria. ${ }^{1-5}$ About 23\% presented with bleed, the rest were incidentally diagnosed on screening endoscopy. The distribution of etiology was similar between bleeders and nonbleeders. GOV1 was less likely to present with bleed. GOV2, on the other hand, presented with bleed more often.

Of all the patients, about one in ten remained uncategorized by the existing system of classification. This classification is based on the location of the varix in the stomach, which ultimately dictates its drainage. Nearly all esophageal varices and GOV1 drain into the azygous system via the periesophageal plexus. ${ }^{11}$ In most cases, GOV2 drains either via the azygous vein to SVC ( $44 \%)$ or via the inferior phrenic vein or gastro-spleno-renal shunt $(\sim 38 \%)$ into the IVC. ${ }^{11,12}$ IGVs drain almost exclusively into the IVC territory, either via the gastrosplenorenal shunt, or in few cases, via the pericardiophrenic (inferior phrenic) veins. ${ }^{13}$

The drawback of the current classification system is that it leaves about a tenth of the GV unclassified. These are often described in the endoscopy report as various combinations of gastric and esophageal varices, which can sometimes be confusing. Moreover, isolated GV may be associated with GOV1 or GOV2 in upto $59 \%$ of cases, rendering the term "isolated gastric varices" technically incorrect. ${ }^{13}$

To avoid this, while retaining the physiological essence of the classification, we suggest that a separate category, GOV3, should be added. This terminology refers to cases where varices are present both in the SVC and IVC draining territories, and covers the previously unclassified cases. Moreover, this will prevent duplicate coding of an individual for epidemiological purposes, especially when a patient exhibits both GOV1/2/esophageal and antral/duodenal varices. Moreover, GOV3 can further be supplemented by the description of location of varices.

The category of GOV3 was able to include the previously uncategorized varices. These varices have two separate drainage systems: those around the gastroesophageal junction have their efferent into the central or right portal system, draining into the SVC via azygous vein. The relatively distal gastric part has efferent into the left portal system, draining via the splenogastrorenal shunt or the pericardiophrenic veins, into the IVC. ${ }^{12}$ GOV3 were three times less likely to present with bleed than other GV. This may have been due to a more effective decompression of the portal system by way of both left and right portal collaterals. Thus, the addition of GOV3 led to complete classification of all varices seen on EGD, while giving some insight on the portal hemodynamics. However, this will have to be confirmed in larger studies.
Due to its retrospective nature, our study was prone to certain biases. Moreover, the lack of follow-up data restricts the information about the natural history and progression of GV. However, we were able to address a lacuna in the categorization of GVs and provide a rational addendum to the nomenclature. We propose that a category GOV3 may be added to the existing Sarin's classification. Addition of GOV3 in Sarin's classification will make it more comprehensive, uniform, and reproducible for future studies. Further prospective studies are needed to discern the outcomes in patients with GOV3 and angiographic studies are required to demonstrate their drainage pathways.

\section{Note}

This work was presented at the annual meeting of The International Liver Congress 2017, Amsterdam, The Netherlands, 19-23 April, 2017.

\section{Authors' Contribution}

Virendra Singh was involved in the development of the protocol and the study, patient enrolment and follow-up, and manuscript writing and data analysis; Akash Singh was involved in data collection and analysis, manuscript writing, and statistical calculations; Nipun Verma was involved in manuscript writing and statistical calculations; Sahaj Rathi was involved in conceptualization, manuscript writing, data analysis, depiction and illustrations; Sunita Kumari was involved in data collection and analysis, manuscript writing, and statistical calculations. Shivani Chandel was involved in manuscript writing. This work conformed to the guidelines laid by Declaration of Helsinki.

\section{Conflict of Interest}

None declared.

\section{Acknowledgments}

None.

\section{References}

1 Sarin SK, Lahoti D, Saxena SP, Murthy NS, Makwana UK. Prevalence, classification and natural history of gastric varices: a long-term follow-up study in 568 portal hypertension patients. Hepatology 1992;16(6):1343-1349

2 Hashizume M, Kitano S, Yamaga H, Koyanagi N, Sugimachi K. Endoscopic classification of gastric varices. Gastrointest Endosc 1990;36(3):276-280

3 Hosking SW, Johnson AG. Gastric varices: a proposed classification leading to management. Br J Surg 1988;75(3):195-196

4 Arakawa M, Masuzaki T, Okuda K. Pathomorphology of esophageal and gastric varices. Semin Liver Dis 2002;22(1):73-82

5 Mathur SK, Dalvi AN, Supe AN, Ramakantan R, Someshwar V. Endoscopic and radiological appraisal of gastric varices. J Gastroenterol Hepatol 1989;4(Suppl 1) :245-247

6 de Franchis R; Baveno VI Faculty. Expanding consensus in portal hypertension: report of the Baveno VI consensus workshop: stratifying risk and individualizing care for portal hypertension. J Hepatol 2015;63(3):743-752

7 Sarin SK. Non-cirrhotic portal fibrosis. Gut 1989;30(3):406-415

8 Sarin SK, Sollano JD, Chawla YK, et al; Members of the APASL Working Party on Portal Hypertension. Consensus on extra-hepatic portal vein obstruction. Liver Int 2006;26(5):512-519 
30 New Classification of Gastric Varices singh et al.

9 Valla DC. Budd-Chiari syndrome/hepatic venous outflow tract obstruction. Hepatol Int 2018;12(Suppl 1):168-180

10 Paquet KJ. Prophylactic endoscopic sclerosing treatment of the esophageal wall in varices - a prospective controlled randomized trial. Endoscopy 1982;14(1):4-5

11 Zhao L-Q, He W, Ji M, Liu P, Li P. 64-row multidetector computed tomography portal venography of gastric variceal collateral circulation. World J Gastroenterol 2010;16(8):1003-1007
12 Saad WE. Vascular anatomy and the morphologic and hemodynamic classifications of gastric varices and spontaneous portosystemic shunts relevant to the BRTO procedure. Tech Vasc Interv Radiol 2013;16(2):60-100

13 Sarin SK, Jain AK, Lamba GS, Gupta R, Chowdhary A. Isolated gastric varices: prevalence, clinical relevance and natural history. Dig Surg 2003;20(1):42-47 